\title{
(2) OPEN ACCESS \\ Stromal MED12 exon 2 mutations in complex fibroadenomas of the breast
}

\author{
Edaise M da Silva (D) ,' Francisco Beca (D) , ${ }^{2}$ Ana Paula Martins Sebastiao, ${ }^{1,3}$ \\ Melissa P Murray, ${ }^{1}$ Catarina Silveira, ${ }^{1,4}$ Arnaud Da Cruz Paula, ${ }^{5}$ Fresia Pareja (D) , \\ Hannah Y Wen, ${ }^{1}$ Timothy M D'Alfonso (D , ' Marcia Edelweiss, ${ }^{1}$ Britta Weigelt, ${ }^{1}$ \\ Edi Brogi, ${ }^{1}$ Jorge $S$ Reis-Filho, ${ }^{1}$ Hong Zhang ${ }^{1}$
}

- Supplemental material is published online only. To view please visit the journal online (http://dx.doi.org/10.1136/ jclinpath-2020-207062).

'Department of Pathology, Memorial Sloan Kettering Cancer Center, New York, NY, USA

${ }^{2}$ Pathology, Stanford University School of Medicine, Stanford,

California, USA

${ }^{3}$ Department of Medical

Pathology, Universidade Federal do Parana Setor de Ciencias da

Saude, Curitiba, Brazil

${ }^{4}$ GenoMed SA, Institute of Molecular Medicine, University of Lisbon, Lisboa, Portugal ${ }^{5}$ Surgery, Memorial Sloan Kettering Cancer Center, New York, NY, USA

\section{Correspondence to} Dr Hong Zhang, Department of Pathology, Memorial Sloan Kettering Cancer Center, New York 10065, New York, USA: zhangh3@mskcc.org

Received 26 August 2020 Revised 23 November 2020 Accepted 7 December 2020

Check for updates

(c) Author(s) (or their employer(s)) 2020. Re-use permitted under CC BY-NC. No commercial re-use. See rights and permissions. Published by BMJ.

To cite: da Silva EM, Beca F, Sebastiao APM, et al. $J$ Clin Pathol Epub ahead of print: [please include Day

Month Year]. doi:10.1136/

jclinpath-2020-207062

\section{ABSTRACT}

Aims Here we explore the presence of mediator complex subunit 12 (MED12) exon 2 and telomerase reverse transcriptase (TERT) promoter hotspot mutations in complex fibroadenomas (CFAs) of the breast.

Methods The stromal components from 18 CFAs were subjected to Sanger sequencing of MED12 exon 2 and the TERT promoter hotspot loci. The epithelial and stromal components of two MED12 mutated CFAs were subjected to laser capture microdissection, and Sanger sequencing of MED12 exon 2, TERT promoter and PIK3CA exons 9 and 20, separately.

Results MED12 exon 2 mutations were identified in the stroma of $17 \%$ of CFAs. The analyses of epithelial and stromal components, microdissected separately, revealed that MED12 mutations were restricted to the stroma. No TERT promoter or PIK3CA mutations in exons 9 and 20 were detected in analysed CFAs.

Conclusions Like conventional fibroadenomas, MED12 exon 2 mutations appear to be restricted to the stromal component of CFAs, supporting the notion that CFAs are stromal neoplasms.

\section{INTRODUCTION}

Fibroadenomas (FAs) of the breast are a group of benign biphasic neoplasms consisting of a proliferation of both epithelial and stromal components. ${ }^{1}$ After the seminal publication by Lim et al, ${ }^{2}$ describing mediator complex subunit 12 (MED12) exon 2 mutations in approximately $60 \%$ of FAs, our group and others have not only confirmed the presence of these mutations in FAs, but also demonstrated that they are restricted to the stromal component of these lesions. ${ }^{2-5}$ MED12 exon 2 mutations have also been detected in other fibroepithelial neoplasms, including phyllodes tumours (PTs), where these mutations are present in $88 \%$ of benign, in $78 \%$ of borderline and in $8 \%$ of malignant PTs. ${ }^{5-8}$ In addition, our group initially described the presence of telomerase reverse transcriptase (TERT) promoter hotspot mutations in PTs, ${ }^{7}$ which have been confirmed to increase in frequency from benign to malignant PTs and to be vanishingly rare in conventional FAs. ${ }^{9-11}$

FAs comprise histologic variants, including juvenile fibroadenoma, cellular FAs and complex fibroadenomas (CFAs), which display different histologic features and variations in their clinicopathologic characteristics. A subset of FAs, characterised by abundant myxoid matrix and hypocellular stromal component, was previously categorised as myxoid fibroadenomas (MFAs) as per fourth edition WHO classification. ${ }^{12}$ These lesions were reported to lack MED12 exon 2 mutations in the stromal component, and to harbour recurrent mutations in TP53, PIK3R1, ERCC5 and PIK3CA in the epithelial rather than the stromal component. ${ }^{13}$ Importantly, in one case of MFA included in Lozada et al, ${ }^{13}$ a mutation affecting PRKAR1A was detected in the stromal component, and the case was subsequently reclassified as a myxoma. Per fifth edition WHO classification of breast neoplasms, ${ }^{1}$ CFA is a variant of fibroadenoma that contains one or more of the following histological findings: cysts $>3 \mathrm{~mm}$, sclerosing adenosis, epithelial calcifications and papillary apocrine metaplasia. As compared with conventional FAs, CFAs have more abundant epithelial components sharing histological features of proliferative fibrocystic disease of breast and less characteristic neoplastic stroma. An increased relative risk (RR) of subsequent invasive breast carcinoma was reported for patients with CFAs (RR 3.10; $95 \%$ CI 1.9 to 5.1) in comparison to patients with conventional FAs (RR 2.17; $95 \%$ CI 1.5 to 3.2). ${ }^{14}$ Other studies, however, have indicated that CFAs may not result in increased breast cancer risk beyond the presence of other established risk factors such as proliferative disease or atypical epithelial hyperplasia of breast. The clinical behaviour of CFAs, however, remains contentious, and the current recommendation is to manage patients with CFA with a conservative approach, similarly to the approach employed for the management of women with conventional FAs. ${ }^{15} 16$

The genetic underpinning of CFAs, whether the abundant epithelial component contributes to the tumorigenesis or whether they are genetically related to conventional FAs or PTs is unclear. Here we conducted an exploratory analysis to determine whether MED12 exon 2 and TERT promoter hotspot mutations would be present in CFAs. Furthermore, given our previous findings that PIK3CA hotspot mutations were present in PTs without FA-like areas and in a subset of FAs, classified as MFAs in the prior (fourth edition) WHO classification, ${ }^{8} 1213$ in addition to the MED12 exon 2 and TERT promoter hotspot mutations, we also assessed the presence of mutations in exons 9 and 20 of PIK3CA in separate epithelial and stromal components of two CFAs subjected to laser capture microdissection (LCM). 


\section{MATERIALS AND METHODS}

\section{Subjects and samples}

Following institutional review board approval, slides and formalin-fixed paraffin-embedded (FFPE) tissue blocks of 24 CFAs from 23 adult patients were retrieved from the archives of the Department of Pathology of Memorial Sloan Kettering Cancer Center (New York, USA). All cases were reviewed by nine breast pathologists (APMS, FP, ME, TMD, MPM, HYW, $\mathrm{EB}$, JSR-F and HZ) and were reclassified according to the WHO criteria fifth edition (2019). ${ }^{1}$ A diagnosis of CFA was rendered when at least one or more complex features were present (ie, cysts $>3 \mathrm{~mm}$, sclerosing adenosis, epithelial calcifications and papillary apocrine metaplasia). Eighteen CFAs (from 17 anonymised patients) were included in this study on a consensus diagnosis being rendered. All cases are unique to this study, and none has been previously reported.

\section{Tumour microdissection and sequencing}

Eight micrometre thick FFPE representative histological sections of 18 CFAs were microdissected under a stereomicroscope (Olympus SZ61) to ensure a stromal cell content $>80 \%$. DNA was extracted using the DNeasy Blood and Tissue Kit (Qiagen) according to the manufacturer's instructions. Sanger sequencing was used for the assessment of mutations affecting MED12 exon 2 and TERT promoter hotspots in the stromal components of 18 CFAs. Sanger sequencing and PCR amplification methods were performed as previously described ${ }^{17}$ and are detailed in online supplemental methods and table S1.

To define whether the mutations affecting the exon 2 of MED12 would be restricted to the stroma or would also be present in the epithelial component in the MED12 mutated cases, representative samples from these tumours were subjected to LCM to obtain the epithelial and stromal components separately (online supplemental methods). Due to the limited material available in one of the cases (CFA7), LCM was performed in two (CFA18 and CFA22) CFAs harbouring MED12 exon 2 mutations. DNA samples from the epithelial and stromal components were subjected to Sanger sequencing of MED12 exon 2, TERT promoter hotspots and PIK3CA exons 9 and 20. Sequence electropherograms of the forward and reverse strands were analysed using Mutation Surveyor (SoftGenetics) and the mutations identified were manually curated. All analyses were performed in duplicate.

\section{RESULTS}

Our study included 18 CFAs from patients with a median age of 48 years at diagnosis (range 33-82 years, (online supplemental table S2). All CFAs harboured at least one complex feature, such as sclerosing adenosis or fibrocystic changes (figure $1 \mathrm{~A}$ and online supplemental table S2). Fibrocystic changes including usual ductal hyperplasia without atypia, apocrine metaplasia, columnar cell changes, cyst formation and stromal fibrosis were identified in all CFAs, and sclerosing adenosis were present in the adjacent breast tissue in $13(72 \%)$ cases. Based on the data retrieved from the pathology reports, concurrent ductal carcinoma in situ (67\%, 12/18), invasive ductal carcinoma (22\%, $4 / 18)$, invasive lobular carcinomas $(6 \%, 1 / 18)$, lobular carcinoma in situ $(11 \%, 2 / 18)$ or atypical ductal hyperplasia $(6 \%, 1 / 18)$ were identified in the breast tissue away from CFAs included in this study.

MED12 exon 2 mutations were identified in the stromal component of 17\% (3/18) of CFAs (figure 1B and online supplemental table S2). Two CFAs harboured the hotspot p.G44S

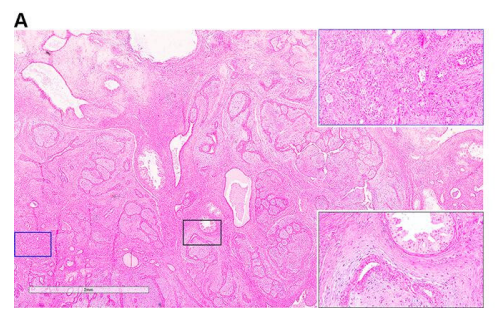

B

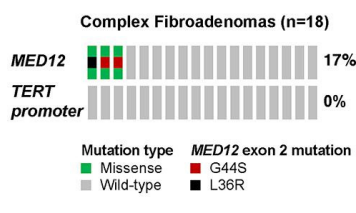

Figure 1 Histologic features and frequency of MED12 exon 2 and TERT promoter mutations in complex FAs of the breast. (A) Representative H\&E micrograph of a complex FA displaying apocrine metaplasia (bottom right) and sclerosing adenosis (top right), scale bar, $2 \mathrm{~mm}$. (B) Frequency of MED12 exon 2 and TERT promoter hotspot mutations in the stromal component of 18 CFAs included in this study. CFAs, complex fibroadenomas; FAs, fibroadenomas; MED12, mediator complex subunit 12; TERT, telomerase reverse transcriptase.

(c.130G $>$ A) and one case harboured the pathogenic p.L36R (c.107T > G) MED12 mutations (figure 2A-C and online supplemental table S3. No differences in the histologic and clinical features of the CFAs, according to the presence or absence of MED12 mutations were found online supplemental figure S1 and table S2. No TERT promoter hotspot mutations were detected in the CFAs analysed (figure 1B, online supplemental table S2).

In CFA18 and CFA22, where the epithelial and stromal components were successfully separated, Sanger sequencing analysis revealed that the MED12 mutations (p.G44S) were exclusively restricted to their respective stromal components. No MED12 mutations were found in the epithelial component of the CFAs (figure 2D and online supplemental table S4). No TERT promoter hotspot mutations or PIK3CA mutations in exons 9 or 20 were found in either the epithelial or stromal component of the CFAs subjected to LCM.

\section{DISCUSSION}

CFAs are a variant of FAs ${ }^{12}$ and comprise $22.7 \%$ to $40.4 \%$ of diagnosed FAs. ${ }^{1418}$ Often clinically overlooked, CFAs tend to occur in patients older than those with conventional FAs ${ }^{15} 18$ with an average size of half of other FAs. ${ }^{15}$ Histologically, the presence of epithelial alterations overlapping with fibrocystic changes inside the lesion is characteristic of this FA subtype. Tubular adenoma of breast, which is a rare epithelial proliferation arising from the terminal duct lobular unit, is occasionally in the differential diagnosis due to the presence of prominent sclerosing adenosis inside the CFA. ${ }^{19}$ Although some studies suggest that CFAs carry a slightly increased risk of subsequent development of breast cancers comparing to general population, ${ }^{14}$ others did not confirm this risk. ${ }^{15} 16$

The exploratory, hypothesis generating analysis performed here revealed that, akin to conventional FAs, a subset of CFAs harbour pathogenic mutations in MED12 exon 2, although at a lower frequency (17\% CFAs vs up to $65 \%$ conventional FAs). ${ }^{5}{ }^{17}$ These findings support the notion that while CFAs and conventional FAs are morphologically and, to a certain extent, clinically separate entities, they share similar genetic alterations in MED12 in a subset of cases, in agreement with what was reported by Lien et al. ${ }^{20}$ Due to the rich nature of epithelial component and its integral growth mixed with stromal components, it is technically challenging to obtain adequate amount of DNA from the separate components in CFAs by LCM. We successfully assessed mutations in MED12 exon 2 and TERT promoter loci in the separately laser microdissected epithelial and stromal components of 

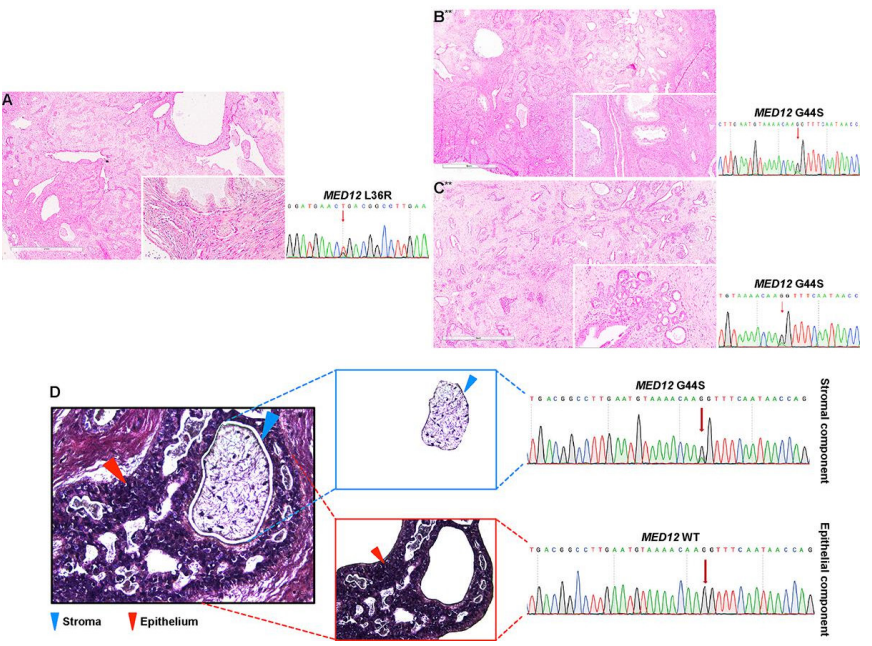

Figure 2 Complex FAs have MED12 exon 2 mutations restricted to the stromal component. Representative H\&E micrographs of CFAs and Sanger sequencing electropherograms of (A) MED12 exon 2 L36R mutation (CFA7), (B-C) MED12 exon 2 G44S mutations (CFA18 and CFA22, respectively) identified, scale bar, $2 \mathrm{~mm}$, (D) CFA18 subjected to LCM and independent sequencing of the epithelial and stromal components (left, blue arrow-stroma, red arrow-epithelium); representative sequence electropherograms of exon 2 of MED12 in the stroma (right top) and the epithelial component (right bottom). ** MED12 mutated CFAs subjected to laser capture microdissection. CFA, complex fibroadenomas; FAs, fibroadenomas; LCM, laser capture microdissection; MED12, mediator complex subunit 12; TERT, telomerase reverse transcriptase.

two CFAs. These cases were also subjected to Sanger analysis of PIK3CA exons 9 and 20. Given that MED12 mutations are restricted to the stromal component, CFAs, akin to conventional FAs, likely constitute stromal rather than fibroepithelial neoplasms. No PIK3CA mutations in exon 9 and 20 were identified in these cases, consistent with previous observations derived from the analysis of fibroepithelial lesions harbouring MED12 mutations. ${ }^{10}$

Our study has limitations. Due to limited archived FFPE tissue availability, we restricted our analysis to specific genetic alterations. Our analysis was restricted to the mutations affecting MED12 exon 2, TERT promoter hotspot mutations, and in two MED12 exon 2 mutated cases, also PIK3CA exons 9 and 20. Although our findings support the notion that MED12 exon 2 mutations do not constitute the main driver of CFAs, we cannot rule out that there are other genes which may play a role in the biology of CFAs. Moreover, due to the size of our cohort and rarity of recurrent events, our study may have a limited power to assess the significance of the presence of MED12 exon 2 mutations in the stromal component of CFAs. All CFAs included in this study were retrieved from the pathology archives of a cancer centre, which might have contributed to the high frequency of co-occurrent invasive and/or in situ carcinomas at the time of the diagnosis of CFA. Given the scope of this study, we could not determine the prognostic significance of MED12 exon 2 mutations and its association with the presence of co-occurrent invasive and/ or in situ carcinomas identified in our cohort. Further studies to assess the impact of MED12 exon 2 mutations in CFAs in the risk of development of breast cancer are warranted. Despite these limitations, here we demonstrated that CFAs harbour MED12 exon 2 mutations in the stromal component, akin to other histologic variants of FAs. At variance with conventional FAs, however, the frequency of MED12 exon 2 mutations in CFAs appears to be lower than that of conventional FAs. Further studies are warranted to define the genetic underpinning of MED12 wild-type CFAs.

Handling editor Cheok Soon Lee.

Contributors FP, EB, JSR-F and $\mathrm{HZ}$ conceived the study. EMdS and $\mathrm{HZ}$ coordinated the retrieval of samples. APMS, MPM, FP, HYW, TMD, ME, EB, JSR-F and HZ performed the pathology review. EMdS, APMS, CS and ADCP performed experiments and analysis. EMdS, FB, BW, JSR-F and HZ analysed and interpreted the data. EMdS, $F B$, JSR-F and $H Z$ wrote the first manuscript, which was reviewed by all coauthors.

Funding This study was funded by the Breast Cancer Research Foundation. BW is funded by a Cycle for Survival grant, CS by a Fundação para a Ciência e Tecnologia grant (SFRH/BDE/110544/2015). FP is partially funded by a K12 CA184746 grant. The research reported in this paper was supported in part by a Cancer Centre Support Grant of the National Institutes of Health/National Cancer Institute (grant No P30CA008748).

Competing interests JSR-F reports receiving personal/consultancy fees from Goldman Sachs and REPARE Therapeutics, membership of the scientific advisory boards of VolitionRx, REPARE Therapeutics and Paige. Al, membership of the Board of Directors of Grupo Oncoclinicas, and ad hoc membership of the scientific advisory boards of Roche Tissue Diagnostics, Ventana Medical Systems, Novartis, Genentech and InVicro, outside the scope of this study. HZ reports consultancy fee from Roche/ Genentech, outside the scope of the submitted work. FB is currently an employee and equity holder of Seattle Genetics, Inc but the present work is outside the scope of his role.

\section{Patient consent for publication Not required.}

Provenance and peer review Not commissioned; externally peer reviewed.

Supplemental material This content has been supplied by the author(s). It has not been vetted by BMJ Publishing Group Limited (BMJ) and may not have been peer-reviewed. Any opinions or recommendations discussed are solely those of the author(s) and are not endorsed by BMJ. BMJ disclaims all liability and responsibility arising from any reliance placed on the content. Where the content includes any translated material, BMJ does not warrant the accuracy and reliability of the translations (including but not limited to local regulations, clinical guidelines, terminology, drug names and drug dosages), and is not responsible for any error and/or omissions arising from translation and adaptation or otherwise.

Open access This is an open access article distributed in accordance with the Creative Commons Attribution Non Commercial (CC BY-NC 4.0) license, which permits others to distribute, remix, adapt, build upon this work non-commercially, and license their derivative works on different terms, provided the original work is properly cited, appropriate credit is given, any changes made indicated, and the use is non-commercial. See: http://creativecommons.org/licenses/by-nc/4.0/.

\section{ORCID iDs}

Edaise M da Silva http://orcid.org/0000-0003-3281-7333

Francisco Beca http://orcid.org/0000-0002-4409-012X

Fresia Pareja http://orcid.org/0000-0003-3748-8049

Timothy M D'Alfonso http://orcid.org/0000-0003-0464-0868

\section{REFERENCES}

1 Thike AA, Brogi E, Harada O, et al. Breast tumours. In: WHO classification of tumors. 5th edn. Lyon: IARC, 2019: 168-71.

2 Lim WK, Ong CK, Tan J, et al. Exome sequencing identifies highly recurrent MED12 somatic mutations in breast fibroadenoma. Nat Genet 2014;46:877-80.

3 Mishima C, Kagara N, Tanei T, et al. Mutational analysis of MED12 in fibroadenomas and phyllodes tumors of the breast by means of targeted next-generation sequencing. Breast Cancer Res Treat 2015;152:305-12.

4 Yoshida M, Sekine S, Ogawa R, et al. Frequent MED12 mutations in phyllodes tumours of the breast. Br J Cancer 2015;112:1703-8.

5 Piscuoglio S, Murray M, Fusco N, et al. MED12 somatic mutations in fibroadenomas and phyllodes tumours of the breast. Histopathology 2015;67:719-29.

6 Tan J, Ong CK, Lim WK, et al. Genomic landscapes of breast fibroepithelial tumors. Nat Genet 2015;47:1341-5

7 Piscuoglio S, Ng CK, Murray M, et al. Massively parallel sequencing of phyllodes tumours of the breast reveals actionable mutations, and TERT promoter hotspot mutations and TERT gene amplification as likely drivers of progression. J Pathol 2016;238:508-18.

8 Pareja F, Geyer FC, Kumar R, et al. Phyllodes tumors with and without fibroadenomalike areas display distinct genomic features and may evolve through distinct pathways. NPJ Breast Cancer 2017;3:40. 
9 Yoshida M, Ogawa R, Yoshida $\mathrm{H}$, et al. TERT promoter mutations are frequent and show association with MED12 mutations in phyllodes tumors of the breast. $\mathrm{Br}$ J Cancer 2015;113:1244-8.

10 Md Nasir ND, Ng CCY, Rajasegaran V, et al. Genomic characterisation of breast fibroepithelial lesions in an international cohort. J Pathol 2019;249:447-60.

11 Garcia-Dios DA, Levi D, Shah V, et al. MED12, TERT promoter and RBM15 mutations in primary and recurrent phyllodes tumours. $\mathrm{Br} J$ Cancer 2018;118:277-84.

12 Lakhani IE SR, Schnitt SJ, Tan PH, et al. WHO classification of breast tumors. 4th edn. Lyon, 2012.

13 Lozada JR, Burke KA, Maguire A, et al. Myxoid fibroadenomas differ from conventional fibroadenomas: a hypothesis-generating study. Histopathology 2017;71:626-34.

14 Dupont WD, Page DL, Parl FF, et al. Long-term risk of breast cancer in women with fibroadenoma. N Engl J Med 1994;331:10-15.

15 Sklair-Levy M, Sella T, Alweiss T, et al. Incidence and management of complex fibroadenomas. AJR Am J Roentgenol 2008;190:214-8.
16 Nassar A, Visscher DW, Degnim AC, et al. Complex fibroadenoma and breast cancer risk: a Mayo clinic benign breast disease cohort study. Breast Cancer Res Treat 2015; 153:397-405.

17 Pareja F, Da Cruz Paula A, Murray MP, et al. Recurrent MED12 exon 2 mutations in benign breast fibroepithelial lesions in adolescents and young adults. J Clin Pathol 2019;72:258-62.

18 Kuijper A, Mommers EC, van der Wall E, et al. Histopathology of fibroadenoma of the breast. Am J Clin Pathol 2001;115:736-42.

19 Volckmar A-L, Leichsenring J, Flechtenmacher C, et al. Tubular, lactating, and ductal adenomas are devoid of MED12 Exon2 mutations, and ductal adenomas show recurrent mutations in GNAS and the PI3K-AKT pathway. Genes Chromosomes Cancer 2017;56:11-17.

20 Lien H-C, Huang C-S, Yang Y-W, et al. Mutational analysis of MED12 exon 2 in a spectrum of fibroepithelial tumours of the breast: implications for pathogenesis and histogenesis. Histopathology 2016;68:433-41. 